\title{
Tonal Scales and Minimal Simple Pitch Class Cycles
}

\author{
David Meredith \\ Aalborg University \\ dave@create.aau.dk
}

\begin{abstract}
Numerous studies have explored the special mathematical properties of the diatonic set. However, much less attention has been paid to the sets associated with the other scales that play an important rôle in Western tonal music, such as the harmonic minor scale and ascending melodic minor scale. This paper focuses on the special properties of the class, $\mathcal{T}$, of sets associated with the major and minor scales (including the harmonic major scale). It is observed that $\mathcal{T}$ is the set of pitch class sets associated with the shortest simple pitch class cycles in which every interval between consecutive pitch classes is either a major or a minor third, and at least one of each type of third appears in the cycle. Employing Rothenberg's definition of stability and propriety, $\mathcal{T}$ is also the union of the three most stable inversional equivalence classes of proper 7-note sets. Following Clough and Douthett's concept of maximal evenness, a method of measuring the evenness of a set is proposed and it is shown that $\mathcal{T}$ is also the union of the three most even 7 -note inversional equivalence classes.
\end{abstract}

Keywords: Diatonic set, Pitch class sets, Pitch class cycles, Scales, Minor scales.

\section{Introduction}

Piston 1] (pp. 1-2) begins his classic textbook on harmony by introducing the chromatic scale and what he calls the "fundamental diatonic scales", that is, the major scale and the natural, harmonic and melodic forms of the minor scale. Each of these scale types is listed in Table 1, together with its associated translational and inversional equivalence classes of pitch class set 1 . Let $\mathcal{F}$ denote the translational equivalence class of pitch class sets associated with Piston's "fundamental diatonic scales". Table 1 shows that $\mathcal{F}=\mathcal{D} \cup \mathcal{H}_{\text {min }} \cup \mathcal{M}$, where $\mathcal{D}$, $\mathcal{H}_{\text {min }}$ and $\mathcal{M}$ are as defined in this table. In this paper, the sets $\mathcal{D}, \mathcal{H}_{\text {min }}$ and $\mathcal{M}$ will be called, respectively, the diatonic sets, the harmonic minor scale sets and the ascending melodic minor scale sets.

Numerous studies have explored the special mathematical properties of the diatonic sets (i.e, $\mathcal{D}$ ) (see [2] for a review). However, as pointed out by Cross

$\left.\overline{{ }^{1}[S]_{\mathrm{T}} \text { and }[S}\right]_{\mathrm{I}}$ (as used in Table 1) denote, respectively, the translational and inversional equivalence class to which pitch class set $S$ belongs.

C. Agon et al. (Eds.): MCM 2011, LNAI 6726, pp. 165 179 2011.

(C) Springer-Verlag Berlin Heidelberg 2011 
Table 1. Piston's "fundamental diatonic scales" along with their associated translational and inversional equivalence classes of pitch class set

\begin{tabular}{l|l|l|l|l} 
Common name & Example & Pitch class set $(P)\left|[P]_{\mathrm{T}}{ }^{a}\right|[P]_{\mathrm{I}}{ }^{a}$ \\
\hline Major & C D E F G A B C & $\{0,2,4,5,7,9,11\}$ & $\mathcal{D}$ & $\mathcal{D}$ \\
Natural minor & A B C D E F G A & $\{9,11,0,2,4,5,7\}$ & $\mathcal{D}$ & $\mathcal{D}$ \\
Harmonic minor & C D Eb F G Ab B C & $\{0,2,3,5,7,8,11\}$ & $\mathcal{H}_{\text {min }}$ & $\mathcal{H}$ \\
Melodic minor ascending & C D Eb F G A B C & $\{0,2,3,5,7,9,11\}$ & $\mathcal{M}$ & $\mathcal{M}$ \\
Melodic minor descending & C Bb Ab G F Eb D C & $\{0,2,3,5,7,8,10\}$ & $\mathcal{D}$ & $\mathcal{D}$ \\
\hline & ${ }^{a} \mathcal{D}=[\{0,1,3,5,6,8,10\}]_{\mathrm{T}}=[\{0,1,3,5,6,8,10\}]_{\mathrm{I}}$ \\
$\mathcal{H}$ & $\mathcal{H}=[\{0,1,3,4,6,8,9\}]_{\mathrm{T}}$ \\
$\mathcal{H}=[\{0,1,3,4,6,8,9\}]_{\mathrm{I}}$ \\
$\mathcal{M}=[\{0,1,3,4,6,8,10\}]_{\mathrm{T}}=[\{0,1,3,4,6,8,10\}]_{\mathrm{I}}$
\end{tabular}

et al. 3] (p. 233), many of these properties are not possessed by the harmonic and ascending melodic forms of the minor scale which clearly "play an extremely significant role in tonal music". In this paper, the focus is on structural properties possessed by all and only those pitch class sets associated with the major and minor scales in all their usual forms.

The sets $\mathcal{D}$ and $\mathcal{M}$ are each closed under inversion. However, the inversion of a harmonic minor scale set is in the transpositional equivalence class $[\{0,1,3,5,6,8,9\}]_{\mathrm{T}}$, denoted here by $\mathcal{H}_{\text {maj }}$. This is the set class associated with what Rahn [4] (p. 41) calls the "major harmonic scales", an example of which is $\mathrm{C}-\mathrm{D}-\mathrm{E}-\mathrm{F}-\mathrm{G}-\mathrm{Ab}-\mathrm{B}-\mathrm{C}$. This scale type has been recognized within tonal theory since at least the mid-19th century. For example, Hauptmann [5] (p. 39) called it the "Moll-Dur-Tonart" (see also McCune [6], pp. 10-11) and Schenker [7] (p. 89) called it the "second series" in his six "products of combination". A cadential progression that ends with a Picardy third often uses all and only the pitch classes in a harmonic major scale - see Fig. 1 .

Let $\mathcal{H}=\mathcal{H}_{\text {min }} \cup \mathcal{H}_{\text {maj. }} \mathcal{H}$ will be called the set of harmonic scale sets. The set $\mathcal{F}$, associated with Piston's "fundamental diatonic scales" is closed under transposition, but not under inversion. The smallest inversionally closed superset

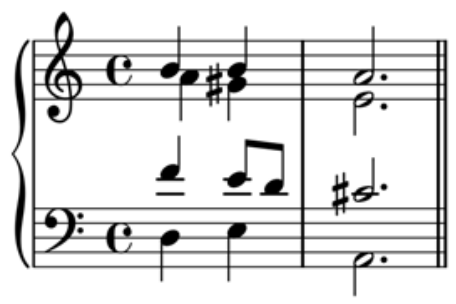

Fig. 1. Example of a Picardy third from the end of J. S. Bach's chorale, Zeuch ein zu deinen Toren (BWV 28/6). This cadential figure employs all and only the pitch classes in a harmonic major scale set. 
of $\mathcal{F}$ is $\mathcal{F} \cup \mathcal{H}_{\text {maj }}$ which will be denoted by $\mathcal{T}$ and called the set of tonal scale sets. $\mathcal{T}$ is therefore given by the following equation.

$$
\mathcal{T}=\mathcal{F} \cup \mathcal{H}_{\text {maj }}=\mathcal{D} \cup \mathcal{M} \cup \mathcal{H}_{\text {min }} \cup H_{\text {maj }}=\mathcal{D} \cup \mathcal{M} \cup \mathcal{H}
$$

Schenker 7] (Chapter 2) describes how six different scales or "series" can be generated from the major and natural minor scales on a given tonic by flattening one or more of the third, sixth and seventh degrees of the major scale (see Table 2). These six "products of combination" along with the major and natural minor scales from which they are generated, transposed to all possible keys, give a set of scales that I'll call Schenker's combination scales. The set of tonal scale sets, $\mathcal{T}$, is the set of pitch class sets associated with Schenker's combination scales (see Table 2). It follows that $\mathcal{T}$ is also the set of pitch class sets associated with scales that can be constructed by combining tonic, dominant and subdominant triads where each can be either major or minor (see Table 2). More tightly, $\mathcal{T}$ is the set of pitch class sets associated with scales that can be constructed by combining a major dominant triad with tonic and subdominant triads that can each be either major or minor (see emboldened lines in Table 2). In other words, $\mathcal{T}$ is the set of sets associated with the major scales and scales constructed by flattening the third and/or sixth degrees in these major scales.

Table 2. Scales with tonic $\mathrm{C}$, generated by combining tonic, dominant and subdominant triads that can be either major or minor. $\mathcal{T}$ is generated by all 8 combinations as well as by only those combinations containing $\mathrm{V}$ (in bold).

\begin{tabular}{|c|c|c|c|c|c|c|}
\hline Triads ${ }^{a}$ & Scale with $C$ as tonic & Pitch class set $(P)$ & {$[P]_{\mathrm{T}}$} & {$[P]_{\mathrm{I}}$} & Mode & Series $^{b}$ \\
\hline I IV V & C D E F A B C & $\{0,2,4,5,7,9,11\}$ & $\mathcal{D}$ & $\mathcal{D}$ & Ionian & - \\
\hline I IV $\mathrm{v}$ & C D E F G A Bb C & $\{0,2,4,5,7,9,10\}$ & $\mathcal{D}$ & $\mathcal{D}$ & Mixolydian & Third \\
\hline I iv $\mathrm{V}$ & $C D E F G A b B C$ & $\{0,2,4,5,7,8,11\}$ & $\mathcal{H}_{\text {maj }}$ & $\mathcal{H}$ & - & Second \\
\hline I iv & C D E F G Ab Bb C & $\{0,2,4,5,7,8,10\}$ & $\mathcal{M}$ & $\mathcal{M}$ & & Sixth \\
\hline i IV V & C D Eb F G A C & $\{0,2,3,5,7,9,11\}$ & $\mathcal{M}$ & $\mathcal{M}$ & & First \\
\hline i IV v & $\mathrm{C} D \mathrm{~Eb} F \mathrm{G} \mathrm{A} \mathrm{Bb} \mathrm{C}$ & $\{0,2,3,5,7,9,10\}$ & $\mathcal{D}$ & $\mathcal{D}$ & Dorian & Fifth \\
\hline i iv $\mathrm{V}$ & C D Eb F G A $b$ B C & $\{0,2,3,5,7,8,11\}$ & $\mathcal{H}_{\text {min }}$ & $\mathcal{H}$ & - & Fourth \\
\hline $\mathrm{i}$ iv & $\mathrm{C}$ D Eb F G Ab Bb C & $\{0,2,3,5,7,8,10\}$ & $\mathcal{D}$ & $\mathcal{D}$ & Aeolian & \\
\hline
\end{tabular}

${ }^{a}$ Upper case denotes a major triad, lower case denotes a minor triad.

${ }^{b}$ Schenker's "series" [7] (pp. 87-93).

\section{Minimal Simple Pitch Class Cycles}

A pitch class sequence is an ordered set of pitch classes. For example, the pitch class sequence $\langle 0,2,4,5,7,9,11,0\rangle$ represents one octave of an ascending $\mathrm{C}$ major scale. Let $\mathbf{S}$ be a pitch class sequence. $|\mathbf{S}|$ denotes the length of $\mathbf{S}$ which is the number of (not necessarily distinct) elements in $\mathbf{S}$. For example $|\langle 0,2,4,2,0\rangle|=$ 5. $\mathbf{S}[i]$ denotes the $(i+1)$ th element in $\mathbf{S}$. For example, $\langle 0,2,4,0\rangle[2]=4 . \mathbf{S}[a, b]$ denotes the subsequence of $\mathbf{S}$ that contains, in order, the $(a+1)$ th to the $b$ th 
elements of $\mathbf{S}$, inclusive. For example, $\langle 0,1,2,3,4,5\rangle[1,4]=\langle 1,2,3\rangle$. If $a=0$ in the expression $\mathbf{S}[a, b]$, then the subsequence is a prefix of $S$. If $b=|S|$, then $\mathbf{S}[a, b]$ is a suffix of $S$. If $\mathbf{A}$ and $\mathbf{B}$ are any two sequences, such that $\mathbf{A}=\left\langle a_{1}, a_{2}, \ldots a_{m}\right\rangle$ and $\mathbf{B}=\left\langle b_{1}, b_{2}, \ldots b_{n}\right\rangle$, then $\mathbf{A} \oplus \mathbf{B}=\left\langle a_{1}, a_{2}, \ldots a_{m}, b_{1}, b_{2}, \ldots b_{n}\right\rangle$. If $\mathbf{S}$ is a sequence, then $\rho(\mathbf{S})$ denotes the reverse of $\mathbf{S}$. That is, $\rho(\mathbf{S})=\langle\mathbf{S}[|\mathbf{S}|-1], \mathbf{S}[|\mathbf{S}|-$ $2], \ldots \mathbf{S}[0]\rangle . \mathrm{P}(\mathbf{S})$ denotes the associated pitch class set of $\mathbf{S}$ which contains all and only those pitch classes in $\mathbf{S}$. That is, $\mathrm{P}(\mathbf{S})=\bigcup_{k=0}^{|\mathbf{S}|-1}\{\mathbf{S}[k]\} . \mathrm{I}(\mathbf{S})$ denotes the interval set of the pitch class sequence $\mathbf{S}$, which is the set of pitch class intervals that occur between consecutive elements in $\mathbf{S}$. For example, $\mathrm{I}(\langle 0,2,4,0\rangle)=\{2,8\}$ and $\mathrm{I}(\langle 0,4,7,11,2,5,9,0\rangle)=\{3,4\} . \mathbf{J}(\mathbf{S})$ denotes the associated interval sequence of the pitch class sequence $\mathbf{S}$ and it is defined as follows:

$$
\mathbf{J}(\mathbf{S})=\langle\mathrm{i}(\mathbf{S}[0], \mathbf{S}[1]), \mathrm{i}(\mathbf{S}[1], \mathbf{S}[2]), \ldots \mathrm{i}(\mathbf{S}[|\mathbf{S}|-2], \mathbf{S}[|\mathbf{S}|-1])\rangle
$$

where $\mathrm{i}\left(p_{1}, p_{2}\right)$ is the pitch class interval from pitch class $p_{1}$ to pitch class $p_{2}$. That is,

$$
\mathrm{i}\left(p_{1}, p_{2}\right)=\left(p_{2}-p_{1}\right) \bmod \mu
$$

where $\mu$ is the chromatic cardinality ([8, p. 94). In this paper, $\mu=12$ unless otherwise stated. From the definitions above, it follows that

$$
\mathrm{I}(\mathbf{S})=\bigcup_{k=0}^{|\mathbf{J}(\mathbf{S})|-1}\{\mathbf{J}(\mathbf{S})[k]\} .
$$

If $\mathbf{S}_{1}$ and $\mathbf{S}_{2}$ are two pitch class sequences, then $\mathbf{S}_{1}$ and $\mathbf{S}_{2}$ are defined to be transpositionally equivalent if and only if $\left|\mathbf{S}_{1}\right|=\left|\mathbf{S}_{2}\right|$ and there exists a single pitch class interval, $i$, such that $\mathrm{i}\left(\mathbf{S}_{1}[k], \mathbf{S}_{2}[k]\right)=i$ for all $0 \leq k<\left|\mathbf{S}_{1}\right|$.

A pitch class cycle is a pitch class sequence that begins and ends on the same pitch class. If $\mathbf{S}$ is a pitch class cycle and $n$ is an integer, then the function $\mathrm{CYC}(\mathbf{S}, n)$ cycles $\mathbf{S}$ by $n$ steps. That is

$$
\mathrm{CYC}(\mathbf{S}, n)=\mathbf{S}[n \bmod (|\mathbf{S}|-1),|\mathbf{S}|] \oplus \mathbf{S}[1, n \bmod (|\mathbf{S}|-1)+1] .
$$

Two pitch class cycles, $\mathbf{S}_{1}$ and $\mathbf{S}_{2}$, are cyclo-transpositionally equivalent if and only if there exists an integer $n$ such that $\mathbf{S}_{1}$ is transpositionally equivalent to $\mathrm{CYC}\left(\mathbf{S}_{2}, n\right)$. A simple pitch class cycle is a pitch class cycle that contains no element more than once except the first element which occurs only at the beginning and at the end. For example $\langle 0,2,4,2,0\rangle$ is a pitch class cycle but it is not simple because 2 occurs twice, and not only at the beginning and the end. $\langle 0,2,4,0\rangle$ is an example of a simple pitch class cycle. A simple pitch class cycle is minimal if and only if there exists no shorter pitch class cycle that has the same interval set. For example, $\langle 0,4,7,11,2,5,9,0\rangle$ is minimal because there exists no shorter pitch class cycle that has the interval set $\{3,4\} . \Gamma(I)$ denotes the set of minimal simple cycles that have the interval set $I . \mathcal{C}(I)$, the set of minimal simple cycle sets for interval set $I$, is defined to contain all and only those pitch class sets associated with cycles in $\Gamma(I)$. 


\section{Minimal Simple Pitch Class Cycles and the Tonal Scale Sets}

It can be shown that $\mathcal{T}$ is the set of minimal simple cycle sets for the interval set $\{3,4\}$, that is,

$$
\mathcal{T}=\mathcal{C}(\{3,4\})
$$

Eq. 6] is important because it expresses a simple mathematical property that is shared by all and only the major and minor scales recognized in traditional tonal theory. It contrasts with those properties of the diatonic set on which recent mathematical work has focused that are not possessed by the common forms of the minor scales. Moreover, Eq. 6] shows how the normal major and minor scales are simply generated by the consonant major and minor third intervals on which tonal harmony is based.

Eq. 6] can be proved by showing separately that $\mathcal{T} \subseteq \mathcal{C}(\{3,4\})$ and that $\mathcal{T} \supseteq \mathcal{C}(\{3,4\})$. Suppose $P \in \mathcal{C}(\{3,4\})$. There therefore exists a minimal simple cycle, $\mathbf{S}$, such that

$$
\begin{aligned}
P & =\mathrm{P}(\mathbf{S}), \\
\mathbf{S}[0] & =\mathbf{S}[|\mathbf{S}|-1], \\
|\mathbf{S}| & =|P|+1, \\
\mathrm{I}(\mathbf{S}) & =\{3,4\} .
\end{aligned}
$$

Moreover, there is no shorter sequence than $\mathbf{S}$ that satisfies Eqs. 7 10, Eq. 10 implies that $\mathbf{J}(\mathbf{S})$ contains $x$ intervals of size 3 and $y$ intervals of size 4 where $x, y \geq 1$. Eq. 9 implies that

$$
x+y=|P| .
$$

Eq. 8 implies that the sum of the intervals in $\mathbf{J}(\mathbf{S})$ is $12 n$ where $n>0$ and $n \in \mathbb{Z}$. Therefore

$$
3 x+4 y=12 n, \text { where } n>0, n \in \mathbb{Z} .
$$

$\mathbf{S}$ is minimal, so $x+y$ must be a minimum. $n=2$ is the smallest value of $n$ that satisfies Eqs. 11 and 12. Therefore $y=3$ and $x=4$. The 3 intervals of size 4 cannot be consecutive in $\mathbf{J}(\mathbf{S})$ as this would either cause a pitch class other than the initial one to be repeated or it would cause the initial pitch class to occur three times. If $\mathbf{J}(\mathbf{S})$ has two consecutive 4 s separated from the third 4 by one interval of size 3 , then $\mathbf{S}$ will be cyclo-transpositionally equivalent to $\langle 0,4,8,11,3,6,9,0\rangle$, implying that $P \in \mathcal{H}_{\text {maj }}$. If the two consecutive 4 s are separated from the third 4 by two $3 \mathrm{~s}$, then $\mathbf{S}$ will be cyclo-transpositionally equivalent to $\langle 0,4,8,11,2,6,9,0\rangle$, implying that $P \in \mathcal{M}$. If the two consecutive 4 s are separated from the third 4 by three $3 \mathrm{~s}$, then $\mathbf{S}$ will be cyclo-transpositionally equivalent to $\langle 0,4,8,11,2,5,9,0\rangle$, implying that $P \in \mathcal{H}_{\text {min }}$. If there are no consecutive intervals of size 4 , then $\mathbf{S}$ will be cyclo-transpositionally equivalent to $\langle 0,4,7,11,2,6,9,0\rangle$, in which case $P \in \mathcal{D}$. This argument is summarised in Table 3. This accounts for all the cyclo-transpositionally distinct possibilities. 
Table 3. Column headed $\mathbf{J}(\mathbf{S})$ shows the five cyclically distinct ways of arranging 3 intervals of size 4 and 4 intervals of size $3 .[\mathrm{P}(\mathbf{S})]_{\mathrm{T}}$ and $[\mathrm{P}(\mathbf{S})]_{\mathrm{I}}$ give the translational and inversional equivalence classes, respectively, associated with the given pitch class cycle.

\begin{tabular}{l|l|l|l}
$\mathbf{J}(\mathbf{S})$ & {$[\mathrm{P}(\mathbf{S})]_{\mathrm{T}}$} & {$[\mathrm{P}(\mathbf{S})]_{\mathrm{I}}$} & Comment \\
\hline$\langle 4,4,4,3,3,3,3\rangle$ & {$[\langle 0,3,4,6,8,9\rangle]_{\mathrm{T}}$} & {$[\langle 0,3,4,6,8,9\rangle]_{\mathrm{I}}$} & Not a simple pitch class cycle. \\
$\langle 4,4,3,4,3,3,3\rangle$ & $\mathcal{H}_{\text {maj }}$ & $\mathcal{H}$ & \\
$\langle 4,4,3,3,4,3,3\rangle$ & $\mathcal{M}$ & $\mathcal{M}$ & \\
$\langle 4,4,3,3,3,4,3\rangle$ & $\mathcal{H}_{\text {min }}$ & $\mathcal{H}$ & \\
$\langle 4,3,4,3,4,3,3\rangle$ & $\mathcal{D}$ & $\mathcal{D}$ & \\
\hline
\end{tabular}

Therefore $P \in \mathcal{C}(\{3,4\}) \Rightarrow P \in \mathcal{H}_{\text {maj }} \cup \mathcal{M} \cup \mathcal{H}_{\text {min }} \cup \mathcal{D}$. Therefore (from Eq. 1) $P \in \mathcal{C}(\{3,4\}) \Rightarrow P \in \mathcal{T}$. Therefore

$$
\mathcal{T} \supseteq \mathcal{C}(\{3,4\})
$$

We now need to show that $\mathcal{T} \subseteq \mathcal{C}(\{3,4\})$. Let $H_{1} \in \mathcal{H}_{\text {maj }}, M \in \mathcal{M}, H_{2} \in \mathcal{H}_{\text {min }}$ and $D \in \mathcal{D}$. There therefore exist simple cycles $\mathbf{H}_{\mathbf{1}}, \mathbf{M}, \mathbf{H}_{\mathbf{2}}$ and $\mathbf{D}$ that are transpositionally equivalent to, respectively, $\langle 0,4,8,11,3,6,9,0\rangle,\langle 0,4,8,11,2,6,9,0\rangle$, $\langle 0,4,8,11,2,5,9,0\rangle$ and $\langle 0,4,7,11,2,6,9,0\rangle$, such that $H_{1}=\mathrm{P}\left(\mathbf{H}_{\mathbf{1}}\right), M=$ $\mathrm{P}(\mathbf{M}), H_{2}=\mathrm{P}\left(\mathbf{H}_{2}\right)$ and $D=\mathrm{P}(\mathbf{D}) . \mathbf{H}_{\mathbf{1}}, \mathbf{M}, \mathbf{H}_{\mathbf{2}}$ and $\mathbf{D}$ each contain 3 intervals of size 4 and 4 intervals of size 3 , therefore $H_{1}, M, H_{2}, D \in \mathcal{C}(\{3,4\})$. Therefore $\mathcal{H}_{\text {maj }} \cup \mathcal{M} \cup \mathcal{H}_{\text {min }} \cup \mathcal{D} \subseteq \mathcal{C}(\{3,4\})$ and so (from Eq. 1) $\mathcal{T} \subseteq \mathcal{C}(\{3,4\})$. This, in conjunction with Eq. 13, implies Eq. 6.

\section{Minimal Simple Cycle Sets and Interval Set Inversion}

If $i$ is a pitch class interval, then the inversion of $i$, denoted $\operatorname{INV}(i)$, is given by $\operatorname{INV}(i)=(-i) \bmod \mu$ where $\mu$ is the chromatic cardinality. If $I$ is a pitch class interval set, then the inversion of $I$ is given by

$$
\operatorname{INV}(I)=\bigcup_{i \in I}\{\operatorname{INV}(i)\}
$$

Lemma 1. If $\mathbf{S}$ is a pitch class sequence, then $\mathrm{I}(\rho(\mathbf{S}))=\operatorname{INV}(\mathrm{I}(\mathbf{S}))$.

Proof. From Eq. 4. INV $(\mathrm{I}(\mathbf{S}))=\operatorname{INV}\left(\bigcup_{k=0}^{|\mathbf{S}|-2}\{\mathrm{i}(\mathbf{S}[k], \mathbf{S}[k+1])\}\right)$. This and Eq. 14 together imply that $\operatorname{INV}(\mathrm{I}(\mathbf{S}))=\bigcup_{k=0}^{|\mathbf{S}|-2}\{\operatorname{INV}(\mathrm{i}(\mathbf{S}[k], \mathbf{S}[k+1]))\}$. Since $\operatorname{INV}\left(i\left(p_{1}, p_{2}\right)\right)=\mathrm{i}\left(p_{2}, p_{1}\right)$, it follows that $\operatorname{INV}(\mathrm{I}(\mathbf{S}))=\bigcup_{k=0}^{|\mathbf{S}|-2}\{\mathrm{i}(\mathbf{S}[k+1], \mathbf{S}[k])\}$ which implies that $\operatorname{INV}(\mathrm{I}(\mathbf{S}))=\mathrm{I}(\rho(\mathbf{S}))$.

Theorem 1. If $I$ is a pitch class interval set, then $\mathcal{C}(I)=\mathcal{C}(\operatorname{INV}(I))$.

Proof. Let $P \in \mathcal{C}(I)$. There therefore exists a minimal simple cycle, $\mathbf{S}$, such that $P=\mathrm{P}(\mathbf{S})$ and $\mathrm{I}(\mathbf{S})=I$. Let $\mathbf{R}=\rho(\mathbf{S})$. From Lemma 1 it follows that $\mathrm{I}(\mathbf{R})=\operatorname{INV}(\mathrm{I}(\mathbf{S}))=\operatorname{INV}(I) . \mathbf{S}$ is a simple cycle, therefore so is $\mathbf{R}$. $\mathbf{R}$ is therefore 
a minimal simple cycle for $\operatorname{INV}(I)$ provided there is no simple cycle shorter than $\mathbf{R}$ whose interval set is $\operatorname{INV}(I)$. We'll suppose $\mathbf{Q} \in \Gamma(\operatorname{INV}(I))$ and that $|\mathbf{Q}|<|\mathbf{R}|$ and show that this leads to a contradiction. $\mathrm{I}(\mathbf{Q})=\operatorname{INV}(I)$, therefore $\mathrm{I}(\rho(\mathbf{Q}))=I$ (from Lemma 1).$\rho(\mathbf{Q})$ is therefore a simple cycle shorter than $\mathbf{R}$ whose interval set is $I$. But $\mathbf{R}$ is the same length as $\mathbf{S}$ so this would mean that $\mathbf{Q}$ is shorter than $\mathbf{S}$, which is impossible because $\mathbf{S}$ is a minimal simple cycle for $I$. Therefore $\mathbf{Q}$ cannot exist and $\mathbf{R} \in \Gamma(\operatorname{INV}(I))$. But $P=\mathrm{P}(\mathbf{S})=\mathrm{P}(\mathbf{R})$, therefore $P \in \mathcal{C}(\operatorname{INV}(I))$ and so $\mathcal{C}(I) \subseteq \mathcal{C}(\operatorname{INV}(I))$. If we now let $J=\operatorname{INV}(I)$, then $\mathcal{C}(J) \subseteq \mathcal{C}(\operatorname{INV}(J))$ and so $\mathcal{C}(\operatorname{INV}(I)) \subseteq \mathcal{C}(I)$. Since $\mathcal{C}(\operatorname{INV}(I)) \subseteq \mathcal{C}(I)$ and $\mathcal{C}(I) \subseteq \mathcal{C}(\operatorname{INV}(I))$, it follows that $\mathcal{C}(I)=\mathcal{C}(\operatorname{INV}(I))$.

Theorem 1 and Eq. 6] together imply that $\mathcal{T}=\mathcal{C}(\{8,9\})$.

\section{Minimal Simple Cycle Sets and the Diatonic Spectra}

A pitch class sequence is simple if and only if it contains no pitch class more than once. If $P$ is a pitch class set, then the associated ascending simple sequence of $P$, denoted $\mathbf{S}(P)$, is the simple pitch class sequence that contains every pitch class in $P$, exactly once, sorted into ascending order. For example, $\mathbf{S}(\{3,2,7,10,8,0,5\})=\langle 0,2,3,5,7,8,10\rangle$. Following Clough and Myerson [9] (p. 262) and Clough and Douthett [8] (p. 95), let the $k$-spectrum, $e_{k}(P)$, of a pitch class set, $P$, be defined as follows:

$$
e_{k}(P)=\{\mathrm{i}(\mathbf{S}(P)[j], \mathbf{S}(P)[(j+k) \bmod |P|])|0 \leq j<| P \mid\},
$$

where $k \in \mathbb{Z}$ and $1 \leq k<|P|$. For example, the 1 -spectrum of $\{0,2,4,5,7,9,11\}$ is $\{1,2\}$ and the 2 -spectrum of the same set (a diatonic set) is $\{3,4\}$.

If $p$ is a pitch class, $i$ is a pitch class interval and $P$ is a pitch class set, then the transposition of $p$ by $i$ is given by $\operatorname{TRAN}(p, i)=(p+i) \bmod \mu$ where $\mu$ is the chromatic cardinality and the transposition of $P$ by $i$ is given by $\operatorname{TRAN}(P, i)=$ $\bigcup_{p \in P}\{\operatorname{TRAN}(p, i)\}$. The following theorem is easy to prove.

Theorem 2. If $P_{1}$ and $P_{2}$ are transpositionally equivalent pitch class sets, then $e_{k}\left(P_{1}\right)=e_{k}\left(P_{2}\right)$ for any given value of $k$.

If $S \subseteq[P]_{\mathrm{T}}$ for some pitch class set $P$, then Theorem 2 allows us to define $e_{k}(S)$ to be the $k$-spectrum common to every pitch class set in $S$ (for some specified value of $k)$. We can therefore state that $e_{2}(\mathcal{D})=\{3,4\}$ and that $e_{5}(\mathcal{D})=$ $\{8,9\}$. In general, $e_{k}(\mathcal{D})$ is the diatonic $k$-spectrum. Therefore, from Eq. 6 and Theorem 1 .

$$
\mathcal{T}=\mathcal{C}\left(e_{2}(\mathcal{D})\right)=\mathcal{C}\left(e_{5}(\mathcal{D})\right) .
$$

If $S \subseteq[P]_{\mathrm{T}}$ for a pitch class set, $P$, then the spectrum set of $S$, denoted by $\mathrm{E}(S)$, is defined to be equal to $\left\{e_{k}(S) \mid 1 \leq k<n\right\}$ where $n$ is the cardinality of a pitch class set in $S . \mathrm{E}(\mathcal{D})$ is therefore the set of diatonic spectra. Table 4 shows the set of minimal simple cycle sets for each diatonic spectrum.

As can be seen in Table 4. $\mathcal{C}\left(e_{1}(\mathcal{D})\right)=\mathcal{C}\left(e_{6}(\mathcal{D})\right)=\mathcal{D} \cup \mathcal{M} \cup \mathcal{W}$, where $\mathcal{W}=$ $[\{0,1,2,4,6,8,10\}]_{\mathrm{I}} \cdot \mathcal{W}$ is the inversional equivalence class of pitch class sets 
Table 4. The set of minimal simple cycle sets for each diatonic spectrum, $\mathcal{C}\left(e_{k}(\mathcal{D})\right)$

\begin{tabular}{l|l|l}
\hline$k$ & $e_{k}(\mathcal{D})$ & $\mathcal{C}\left(e_{k}(\mathcal{D})\right)^{a}$ \\
\hline 1 & $\{1,2\}$ & $\mathcal{D} \cup \mathcal{M} \cup \mathcal{W}$ \\
2 & $\{3,4\}$ & $\mathcal{D} \cup \mathcal{M} \cup \mathcal{H}$ \\
3 & $\{5,6\}$ & $\mathcal{D}$ \\
4 & $\{6,7\}$ & $\mathcal{D}$ \\
5 & $\{8,9\}$ & $\mathcal{D} \cup \mathcal{M} \cup \mathcal{H}$ \\
6 & $\{10,11\}$ & $\mathcal{D} \cup \mathcal{M} \cup \mathcal{W}$ \\
\hline
\end{tabular}

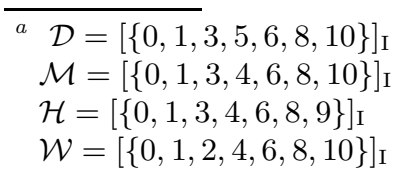

associated with what Rahn 4] (p. 37) calls the "neapolitan ascending melodic minor scale" or "whole tone scale with filler tone". Table 4 also illustrates the following lemma.

Lemma 2. If $S \subseteq[P]_{\mathrm{T}}$ for some specified pitch class set, $P$, then $e_{k}(S)=$ $\operatorname{INV}\left(e_{n-k}(S)\right)$ where $1 \leq k<n$ and $n=|P|$.

Proof By definition, INV $\left(e_{n-k}(S)\right)=\operatorname{INV}(\{\mathrm{i}(\mathbf{S}(P)[m], \mathbf{S}(P)[(m+n-k) \bmod$ $n]) \mid 0 \leq m<n\})$. Therefore $\operatorname{INV}\left(e_{n-k}(S)\right)=\operatorname{INV}(\{\mathrm{i}(\mathbf{S}(P)[m], \mathbf{S}(P)[(m-$ $k) \bmod n]) \mid 0 \leq m<n\})=\{\mathrm{i}(\mathbf{S}(P)[(m-k) \bmod n], \mathbf{S}(P)[m]) \mid 0 \leq m<n\}$ where $1 \leq k<n$. Let $j=(m-k) \bmod n$ so that $(j+k) \bmod n=m$. It follows that $\operatorname{INV}\left(e_{n-k}(S)\right)=\{\mathrm{i}(\mathbf{S}(P)[j], \mathbf{S}(P)[(j+k) \bmod n]) \mid 0 \leq j<n\}=e_{k}(S)$ where $1 \leq k<n$.

We can then infer the following theorem, also illustrated in Table 4 .

Theorem 3. If $S \subseteq[P]_{\mathrm{T}}$ for some specified pitch class set, $P$, then $\mathcal{C}\left(e_{k}(S)\right)=$ $\mathcal{C}\left(e_{n-k}(S)\right)$ where $1 \leq k<n$ and $n=|P|$.

Proof From Theorem 1 we know that $\mathcal{C}\left(e_{k}(S)\right)=\mathcal{C}\left(\operatorname{INV}\left(e_{k}(S)\right)\right)$. But Lemma 2 tells us that $e_{k}(S)=\operatorname{INV}\left(e_{n-k}(S)\right)$. Therefore $\mathcal{C}\left(e_{k}(S)\right)=$ $\mathcal{C}\left(\operatorname{INV}\left(\operatorname{INV}\left(e_{n-k}(S)\right)\right)\right)$. Therefore $\mathcal{C}\left(e_{k}(S)\right)=\mathcal{C}\left(e_{n-k}(S)\right)$.

If $S \subseteq[P]_{\mathrm{T}}$ for some specified pitch class set, $P$, then let $\mathcal{C}(S)=\bigcup_{e \in \mathrm{E}(S)} \mathcal{C}(e)$. As can be seen in Table 4 .

$$
\mathcal{C}(\mathcal{D})=\mathcal{D} \cup \mathcal{M} \cup \mathcal{H} \cup \mathcal{W}=\mathcal{T} \cup \mathcal{W}
$$

In other words, the set of minimal simple cycle sets associated with the diatonic spectra is equal to the union of the set of tonal scale sets, $\mathcal{T}$, and the set of sets associated with what Rahn [4] (p. 37) calls the "whole-tone scale with filler tone", $\mathcal{W}$. 


\section{$6 \mathcal{C}(\mathcal{D})$ and Contradictions}

The concept of a contradiction discussed by Rahn [4] (p. 36) and Clough and Douthett [8] (p. 125) can be defined as follows.

Definition 1. If $P$ is a pitch class set and there exist integers, $j, k, \ell$ and $m$ such that $\mathrm{i}(\mathbf{S}(P)[\ell], \mathbf{S}(P)[m])>\mathrm{i}(\mathbf{S}(P)[j], \mathbf{S}(P)[k])$ and $(m-\ell) \bmod |P|<$ $(k-j) \bmod |P|$, then this is a case of contradiction.

Let a non-contradictory set be one that has no contradictions. Let $\mathcal{R}_{\mu, n}$ denote the set of non-contradictory sets of cardinality $n$ when the chromatic cardinality is $\mu$. Rahn [4] (p. 37) observes that $\mathcal{R}_{12,7}=\mathcal{D} \cup \mathcal{M} \cup \mathcal{H} \cup \mathcal{W}$. From Eq. 17] above, it follows that $\mathcal{C}(\mathcal{D})=\mathcal{R}_{12,7}$. That is, a set is a minimal simple cycle set for a diatonic spectrum if and only if it is a non-contradictory set of cardinality 7 .

\section{The Anhemitonic Pentatonic Scale and the Major Dominant Ninth Chord}

An interval set $I$ is contiguous if and only if there exist $j$ and $k$ such that $I=\{i \mid j \leq i<k\}$. Each diatonic spectrum is a contiguous set of the form $\{i, i+1\}$ where $i \in\{1,3,5,6,8,10\}$ (see Table 4). Each of these spectra has an interesting set of minimal simple cycle sets associated with it, which makes one wonder what the minimal simple cycle sets might be for interval sets of the form $\{i, i+1\}$ where $i \notin\{1,3,5,6,8,10\}$-that is, where $i \in\{2,4,7,9\}$. This set of contiguous-pair interval sets is equal to the spectrum set of the usual, "black note", anhemitonic pentatonic scale, $\{\mathrm{F} \sharp, \mathrm{G} \sharp, \mathrm{A} \sharp, \mathrm{C} \sharp, \mathrm{D} \sharp\}$, that is, $\mathrm{E}(\mathcal{P})=$ $\{\{2,3\},\{4,5\},\{7,8\},\{9,10\}$,$\} where \mathcal{P}=[\{0,2,4,7,9\}]_{\mathrm{T}}=[\{0,2,4,7,9\}]_{\mathrm{I}}$. Let us call $\mathrm{E}(\mathcal{P})$ the set of pentatonic spectra and $e_{k}(\mathcal{P})$ the pentatonic $k$-spectrum. It can readily be shown that $\mathcal{C}\left(e_{2}(\mathcal{P})\right)=\mathcal{C}\left(e_{3}(\mathcal{P})\right)=\mathcal{P}$ and that $\mathcal{C}\left(e_{1}(\mathcal{P})\right)=$ $\mathcal{C}\left(e_{4}(\mathcal{P})\right)=\mathcal{P} \cup \mathcal{N}$ where $\mathcal{N}=[\{0,2,4,6,9\}]_{\mathrm{T}}=[\{0,2,4,6,9\}]_{\mathrm{I}} \cdot \mathcal{N}$ is the set of sets associated with the complete major dominant ninth chords (see, e.g., Piston [1, pp. 334-346). It therefore follows that $\mathcal{C}(\mathcal{P})=\mathcal{P} \cup \mathcal{N}$.

There are intriguing parallels between the sets of minimal simple cycle sets for the diatonic spectra and those for the pentatonic spectra. First, for $k$-spectra where $k \in\{\lfloor|P| / 2\rfloor,\lceil|P| / 2\rceil\}$, the minimal simple cycle sets are diatonic if $P$ is diatonic and pentatonic if $P$ is pentatonic. For the other spectra, the minimal simple cycle sets include other scale or chord types as well as the diatonic /pentatonic sets: for the other diatonic spectra, the other scale types generated are the harmonic major and minor, the ascending melodic minor and the "whole tone plus filler tone" scales; for the other pentatonic spectra, the other set class that emerges is that associated with the complete major dominant ninth chord. From the perspective of minimal simple cycle sets, the relationship between the major dominant ninth set and the pentatonic scale set is analogous to that between the diatonic set and the minor scales. Moreover, just as the set complement of a diatonic set $(\mathcal{D})$ is a pentatonic scale set $(\mathcal{P})$, so the set complement of an ascending melodic minor scale set $(\mathcal{M})$ is a major dominant ninth $\operatorname{set}(\mathcal{N})$. 


\section{$8 \mathcal{C}(\mathcal{D}), \mathcal{C}(\mathcal{P})$ and Rothenberg's Concept of Propriety}

Let $x, y, z, w, k$ and $\ell$ be any six tones on a continuous frequency scale, $S$, and let $(h j)$ denote the undirected interval between tones $h$ and $j$. Rothenberg [10] (pp. 206-207) defines a function $F(h j)$ that maps all musical intervals, $(h j) \in$ $S \times S$, onto a set of discrete points called the code. Such a mapping is defined to be proper if and only if

$$
(F(x y)=F(z w)) \wedge((x y)<(k l)<(z w)) \Rightarrow(F(k l)=F(x y)) .
$$

Rothenberg defines a contradiction to be any violation of this condition (i.e., $F(k l)>F(z w)$ or $F(k l)<F(x y))$. If $S$ is a discrete set of pitches (e.g., the ones that can be produced on a normal piano) and we define a specific interval to be one between pitches in this discrete $S$ and a generic interval to be the code onto which each specific interval is mapped, then a contradiction in Rothenberg's sense occurs when the ordering of two specific intervals is the opposite of the ordering of their corresponding generic intervals. The concept of contradiction defined in the more recent papers of Rahn [4] (p. 36) and Clough and Douthett [8] (p. 125) is therefore identical to Rothenberg's concept in the special case where $S$ is a discrete set of pitch points.

Rothenberg [10] (pp. 206-7) defines a mapping $F$ to be strictly proper if and only if it satisfies Eq. 18 and

$$
(x y) \sim(z w) \Rightarrow F(x y)=F(z w)
$$

where $(x y) \sim(z w)$ means that the intervals $(x y)$ and $(z w)$ sound the same. Thus, for a mapping to be strictly proper, the range of intervals that maps onto a given code must be contiguous and must not overlap with the range of intervals that map onto some other code. If Eq. 19 is not satisfied (i.e., two intervals, that sound the same, map onto different codes), then Rothenberg calls this an ambiguity. In the special case where $S$ is discrete, Rothenberg's concept of ambiguity is therefore identical to that of Rahn [4] (p. 36) and Clough and Douthett [8] (p. 125), despite the fact that neither of these more recent papers attributes the concept to Rothenberg.

Rothenberg [10] (p. 207) defines an ambiguous interval to be one that is mapped by $F$ onto more than one code. If $P$ is the range of $F$, then the stability of $P$ is the ratio of the number of non-ambiguous undirected intervals in $P$ to the total number of undirected intervals [11] (p. 354). For example, the stability of the diatonic set is $\frac{20}{21}$. If $P$ and $Q$ are pitch class sets, then (following Rothenberg [11, p. 369) $Q$ is a sufficient set for $P$ if and only if $P$ is the only element of $[P]_{\mathrm{T}}$ that contains $Q$; and $Q$ is a minimal sufficient set for $P$ if $Q$ is a sufficient set for $P$ and does not contain any proper subset which is also a sufficient set for $P$. Let $\mathbf{S}_{i}$ be any pitch class sequence that contains every element of $P$ exactly once. There are therefore $(|P|)$ ! distinct sequences $\mathbf{S}_{i}$. Now let $s_{i}$ be the length of the shortest prefix of $\mathbf{S}_{i}$ whose associated pitch class set is a sufficient set for $P$. The average value of $s_{i}$ over all sequences $\mathbf{S}_{i}$ for a pitch class set $P$ is given by $\sigma(P)=\left(\sum_{i} s_{i}\right) /(|P| !)$ and Rothenberg [1] (p. 370) defines the efficiency of 
Table 5. The inversional equivalence classes that contain proper 5- and 7-note sets for $\mu=12$, together with the stability and efficiency of the sets in each class. (From Rothenberg [1], p. 367).

\begin{tabular}{l|l|l|l}
\hline$P$ & {$[P]_{\mathrm{I}}$} & Stability & Efficiency \\
\hline$\{0,1,4,6,8\}$ & {$[\{0,1,4,6,8\}]_{\mathrm{I}}$} & 0.6000 & 0.5800 \\
$\{0,2,4,6,8\}$ & {$[\{0,2,4,6,8\}]_{\mathrm{I}}$} & 0.4000 & 1.0000 \\
$\{0,2,4,7,9\}$ & $\mathcal{P}=[\{0,2,4,7,9\}]_{\mathrm{I}}$ & 1.0000 & 0.8000 \\
$\{0,1,4,6,9\}$ & {$[\{0,1,4,6,9\}]_{\mathrm{I}}$} & 0.9000 & 0.6000 \\
$\{0,2,4,6,9\}$ & $\mathcal{N}=[\{0,2,4,6,9\}]_{\mathrm{I}}$ & 0.9000 & 0.6400 \\
$\{0,1,3,6,9\}$ & {$[\{0,1,3,6,9\}]_{\mathrm{I}}$} & 0.4000 & 0.6400 \\
$\{0,1,3,4,6,8,9\}$ & $\mathcal{H}=[\{0,1,3,4,6,8,9\}]_{\mathrm{I}}$ & 0.4762 & 0.6259 \\
$\{0,1,3,5,6,8,10\}$ & $\mathcal{D}=[\{0,1,3,5,6,8,10\}]_{\mathrm{I}}$ & 0.9542 & 0.7687 \\
$\{0,1,3,4,6,8,10\}$ & $\mathcal{M}=[\{0,1,3,4,6,8,10\}]_{\mathrm{I}}$ & 0.7143 & 0.6299 \\
$\{0,1,2,4,6,8,10\}$ & $\mathcal{W}=[\{0,1,2,4,6,8,10\}]_{\mathrm{I}}$ & 0.2857 & 0.6327 \\
\hline
\end{tabular}

$P$ to be $\varepsilon(P)=\sigma(P) /|P|$. The efficiency of a set $P$ is therefore the average size of a sufficient set for $P$ as a proportion of the size of $P$. It indicates how much of a set needs to be presented on average in order for it to be distinguished from its transpositions.

Table 5 lists every proper inversional equivalence class of 5- and 7-note sets for a chromatic modulus of 12 , together with its stability and efficiency. Note that a proper set is strictly proper if and only if its stability is 1 . This table reveals a number of interesting facts. First, if we rank the inversional equivalence classes of proper 5-note sets into decreasing order by, first, stability and then efficiency, then the union of the two highest-ranked classes is equal to $\mathcal{C}(\mathcal{P})=\mathcal{P} \cup \mathcal{N}$. Next, the set of proper 7 -note sets is equal to $\mathcal{C}(\mathcal{D})=\mathcal{R}_{12,7}$. Finally, the union of the three most stable inversional equivalence classes of proper 7 -note sets is equal to $\mathcal{T}$, the set of tonal scale sets. We can speculate that the rather low stability of $\mathcal{W}$ may be related to the fact that it seems to have been used much less commonly by composers than the other minimal simple cycle sets for the diatonic spectra.

\section{$9 \mathcal{T}, \mathcal{C}(\mathcal{P})$ and Carey's Coherence Quotient}

Like Balzano 12] (p. 71), Carey 13. (p. 6) defines a set to be coherent only if it contains neither contradictions nor ambiguities (in the sense of Rothenberg [10], p. 207, discussed above). Let $S$ be a pitch class set of cardinality $N$. Carey denotes by $A F(S)$ the total number of coherence failures in $S$-that is, the total number of contradictions and ambiguities in $S$ [13 (p. 19). Carey also denotes by $S F(N)$ the maximum number of simultaneous failures for a set of cardinality $N$ 13] (p. 14). He then defines the coherence quotient of $S$, denoted by $C Q(S)$, to be $1-(A F(S) / S F(N))$ and proposes that sets with higher coherence quotients will be more "scale-like" [13] (p. 19). Carey shows that the three, 7note, inversional equivalence classes with the highest coherence quotients are (in decreasing order) $[\{0,1,3,5,6,8,10\}]_{\mathrm{I}}($ i.e., $\mathcal{D}),[\{0,1,3,4,6,8,10\}]_{\mathrm{I}}$ (i.e., $\left.\mathcal{M}\right)$ 
and $[\{0,1,3,4,6,8,9\}]_{\mathrm{I}}($ i.e., $\mathcal{H})[13]$ (p. 36). In other words, the set of tonal scale sets, $\mathcal{T}$, is equal to the union of the three, 7 -note, inversional equivalence classes with the highest coherence quotients. When we consider the most coherent 5note sets, we find one inversional equivalence class, $\mathcal{P}=[\{0,2,4,7,9\}]_{\mathrm{I}}$, with a maximal coherence quotient of 1 and then two classes, $\mathcal{N}=[\{0,2,4,6,9\}]_{\mathrm{I}}$ and $[\{0,1,4,6,9\}]_{\mathrm{I}}$ with a coherence quotient of $0.96[13$ (p. 42). The $\operatorname{set} \mathcal{C}(\mathcal{P})$ is therefore a subset of the union of the 5-note classes with the two highest coherence quotients.

\section{Measuring the Evenness of a Pitch Class Set}

Clough and Douthett [8] (p. 96) define a set to be maximally even if and only if every spectrum of the set is either a single integer or a pair of consecutive integers. However, they do not suggest how the evenness of a set might be measured.

Figure 2 represents the diatonic set $\{0,1,3,5,6,8,10\}$. Each pitch class in the set is connected to the centre of the diagram by a radial line segment. The arcs represent springs that start and end on these radial line segments. Suppose all the springs actually connect to the radial line segments at the same distance from the centre (despite the way the diagram is drawn). Suppose also that every spring is constrained in some frictionless way to lie on the circumference of a circle whose centre is at the centre of the diagram. Assume each spring obeys Hooke's law and that they all have the same spring constant $k$. The potential energy stored in a spring is $u(\Delta x)=\frac{k}{2}(\Delta x)^{2}$, where $\Delta x$ is the difference between the length of the spring and its resting length. A spring is defined to be at its resting length when the angle between the two pitch classes that it connects is the angle that would be between them if all the pitch classes in the set were perfectly evenly distributed around the circle.

If $p_{1}$ and $p_{2}$ are two pitch classes in a pitch class set $P$, then let the diatonic interval, $d\left(p_{1}, p_{2}, P\right)$, be defined to be $\left(\operatorname{POS}\left(p_{2}, \mathbf{S}(P)\right)-\operatorname{POS}\left(p_{1}, \mathbf{S}(P)\right)\right) \bmod |P|$ where $\operatorname{POS}(x, \mathbf{S})$ is the index of the first occurrence of $x$ in $\mathbf{S}$ (or null if $x$ is not in $\mathbf{S}$ ). For a pitch class set $P$, the resting length of the spring connecting $p_{1}$ and $p_{2}$ is $2 \pi r d /|P|$ where $d$ is the diatonic interval associated with the lesser of $\mathrm{i}\left(p_{1}, p_{2}\right)$ and $\mathrm{i}\left(p_{2}, p_{1}\right)$ and $r$ is the distance from the centre to the spring. If we suppose that $r=1 /(2 \pi)$, then the resting spring length reduces to $d /|P|$ and the actual spring length is equal to $c / \mu$ where $c$ is the smaller of $\mathrm{i}\left(p_{1}, p_{2}\right)$ and $\mathrm{i}\left(p_{2}, p_{1}\right) . \Delta x$ for a spring is therefore $\frac{c}{\mu}-\frac{d}{|P|}$ and the potential energy stored in the spring is $k\left(\frac{c}{\mu}-\frac{d}{|P|}\right)^{2} / 2$, which reduces to $((c / \mu)-(d /|P|))^{2}$ if we set $k=2$. We can use this potential energy as a measure of the "unevenness" within $P$ of the interval between the two pitch classes connected by the spring. We can then sum the potential energies of all the springs to get a measure of the unevenness of $P$. Formally, if $P$ is a pitch class set and $p_{1}, p_{2} \in P$, then the unevenness associated with the pair of pitch classes $p_{1}$ and $p_{2}$ is

$$
u\left(p_{1}, p_{2}, P\right)=\left(\left(\gamma\left(p_{1}, p_{2}\right) / \mu\right)-\left(\delta\left(p_{1}, p_{2}, P\right) /|P|\right)\right)^{2}
$$




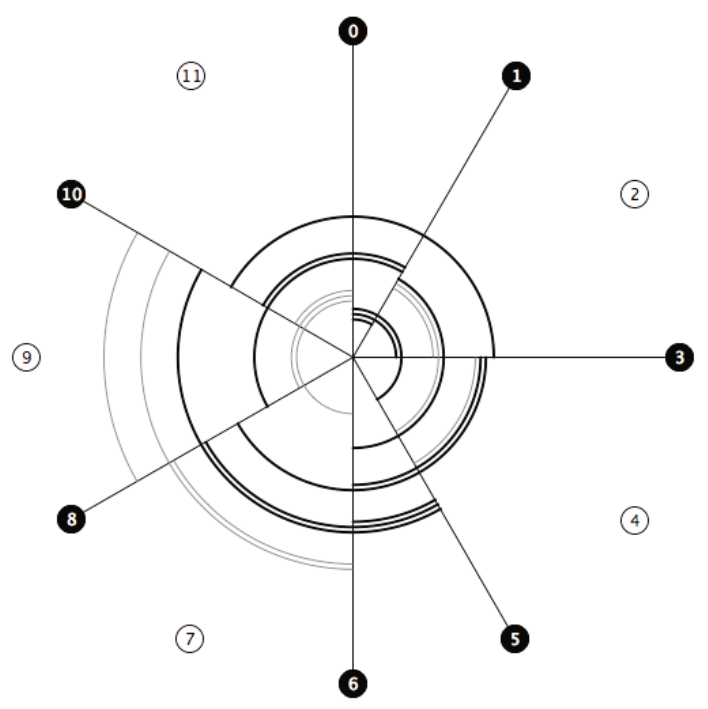

Fig. 2. Representation of the diatonic set illustrating a proposed method of measuring evenness. Each arc represents a spring connecting the pitch classes whose radial line segments intersect the endpoints of the arc. Stretched springs are thin and grey, compressed springs are thick and black.

where $\gamma\left(p_{1}, p_{2}\right)=\min \left(\mathrm{i}\left(p_{1}, p_{2}\right), \mathrm{i}\left(p_{2}, p_{1}\right)\right)$ and

$$
\delta\left(p_{1}, p_{2}, P\right)= \begin{cases}d\left(p_{1}, p_{2}, P\right) & \text { if } \mathrm{i}\left(p_{1}, p_{2}\right)<\mathrm{i}\left(p_{2}, p_{1}\right), \\ d\left(p_{2}, p_{1}, P\right) & \text { if } \mathrm{i}\left(p_{1}, p_{2}\right)>\mathrm{i}\left(p_{2}, p_{1}\right), \\ \min \left(d\left(p_{1}, p_{2}, P\right), d\left(p_{2}, p_{1}, P\right)\right) & \text { if } \mathrm{i}\left(p_{1}, p_{2}\right)=\mathrm{i}\left(p_{2}, p_{1}\right) .\end{cases}
$$

The unevenness of the set $P$ is then given by

$$
U(P)=\sum_{i=0}^{|P|-2}\left(\sum_{j=i+1}^{|P|-1} u(\mathbf{S}(P)[i], \mathbf{S}(P)[j], P)\right) .
$$

When the unevenness of a set is measured in this way, it turns out that: (1) the set of non-singleton, perfectly even sets (i.e., the ones that have an unevenness of 0 and a cardinality greater than 1 ) is equal to the set of minimal simple cycle sets for interval sets of cardinality $1 ;(2) \mathcal{T}$ is equal to the union of the three most even 7 -note inversional equivalence classes; and $(3) \mathcal{C}(\mathcal{P})$ is equal to the union of the two most even 5 -note inversional equivalence classes.

Note that $\mathcal{T}$ is not the union of the three most even 7 -note inversional equivalence classes when certain other measures that have been proposed in the literature are used to measure evenness. Table 6 shows the 7 -note set classes that are judged to be most even using Eq. 21 along with their ranks using Block and 
Table 6. Most even 7-note set classes when measured using Eq.21 with ranks generated by Block and Douthett's [14] (pp. 32-35) measure for comparison

\begin{tabular}{l|l|l|l} 
Rank using Eq. [21 & $U(P)$ & {$[P]_{\mathrm{I}}$} & Block and Douthett's rank \\
\hline 1 & 0.0278 & $\mathcal{D}=[\{0,1,3,5,6,8,10\}]_{\mathrm{I}}$ & 1 \\
2 & 0.0417 & $\mathcal{M}=[\{0,1,3,4,6,8,10\}]_{\mathrm{I}}$ & 2 \\
3 & 0.0556 & $\mathcal{H}=[\{0,1,3,4,6,8,9\}]_{\mathrm{I}}$ & 4 \\
$4=$ & 0.0694 & $\mathcal{W}=[\{0,1,2,4,6,8,10\}]_{\mathrm{I}}$ & 3 \\
$4=$ & 0.0694 & {$[\{0,1,2,4,6,8,9\}]_{\mathrm{I}}$} & $?$ \\
\hline
\end{tabular}

Douthett's measure [14] (pp. 32-35). Note that $\mathcal{C}(\mathcal{D})$ is a subset of the union of the set classes with the highest 4 ranks when evenness is calculated with either measure. However, $\mathcal{T}$ is no longer the union of the three most even 7 -note classes when Block and Douthett's measure is used. This may suggest that the measure defined in Eq. 21 is a better model of intuition than that proposed by Block and Douthett, a hypothesis that could be investigated further by carrying out appropriate psychological experiments.

\section{Summary of Main Results}

The set $\mathcal{F}=\mathcal{D} \cup \mathcal{M} \cup \mathcal{H}_{\text {min }}$ is the set of pitch class sets associated with what Piston 1] (pp. 1-2) calls the "fundamental diatonic scales". The smallest inversionally closed superset of $\mathcal{F}$ is the set $\mathcal{T}=\mathcal{F} \cup \mathcal{H}_{\text {maj }}$ where $\mathcal{H}_{\text {maj }}$ is the set of sets associated with the harmonic major scales. In this paper, $\mathcal{T}$ is called the set of tonal scale sets as it contains all and only those sets associated with the usual major and minor scales. It has been shown that the set $\mathcal{T}$ is equal to:

1. the smallest inversionally closed superset of $\mathcal{F}$;

2. the set of pitch class sets associated with Schenker's combination scales;

3. the set of pitch class sets generated by combining a major dominant triad with a major or minor tonic triad and a major or minor subdominant triad;

4. the set of pitch class sets generated by combining primary triads that can each be either major or minor;

5. the set of minimal simple cycle sets for the diatonic 2-spectrum, $\{3,4\}$;

6 . the union of the three most stable inversional equivalence classes of proper 7-note sets (using Rothenberg's [1011] definitions of stability and propriety);

7. the union of the three, 7-note inversional equivalence classes with the highest coherence quotient (as defined by Carey [13]); and

8. the union of the three most even 7-note inversional equivalence classes (using the spring-based method of measuring evenness proposed in Sect. 10).

It was also shown that the set of non-contradictory 7 -note sets, $\mathcal{R}_{12,7}$ is equal to the set $\mathcal{C}(\mathcal{D})$, that is, the union of the sets of minimal simple cycle sets for the diatonic spectra.

Finally, it was shown that the union of the sets of minimal simple cycle sets for the pentatonic spectra, $\mathcal{C}(\mathcal{P})$, is equal to $\mathcal{P} \cup \mathcal{N}$ where $\mathcal{N}$ is the class of 
pitch class sets associated with the complete major dominant ninth chord. The relationship between $\mathcal{N}$ and $\mathcal{P}$ is therefore analogous to that between $\mathcal{D}$ and the other tonal scales. $\mathcal{C}(\mathcal{P})$ is also:

1. the union of the two most highly-ranked inversional equivalence classes of proper 5-note sets when these are sorted by stability and then efficiency;

2. a subset of the union of the 5-note inversional equivalence classes with the two highest coherence quotients (as defined by Carey [13]); and

3 . the union of the two most even 5-note inversional equivalence classes (when evenness is measured using the spring-based method proposed in Sect. 10).

\section{References}

1. Piston, W.: Harmony. Gollancz, London (1991)

2. Clough, J., Engebretsen, N., Kochavi, J.: Scales, Sets and Interval cycles: A Taxonomy. Music Theory Spectrum 21(1), 74-104 (1999)

3. Cross, I., West, R., Howell, P.: Cognitive Correlates of Tonality. In: Howell, P., West, R., Cross, I. (eds.) Representing Musical Structure, pp. 201-243. Academic Press, London (1991)

4. Rahn, J.: Coordination of Interval Sizes in Seven-Tone Collections. Journal of Music Theory 35(1/2), 33-60 (1991)

5. Hauptmann, M.: Die Natur der Harmonik und der Metrik zur Theorie der Musik. Breitkopf und Härtel, Leipzig (1853)

6. McCune, M.: Moritz Hauptmann: Ein haupt Mann in Nineteenth Century Music Theory. Indiana Theory Review 7(2), 1-28 (1986)

7. Schenker, H.: Harmony. University of Chicago Press, Chicago (1954)

8. Clough, J., Douthett, J.: Maximally Even Sets. Journal of Music Theory 35(1/2), 93-173 (1991)

9. Clough, J., Myerson, G.: Variety and Multiplicity in Diatonic Systems. Journal of Music Theory 29(2), 249-270 (1985)

10. Rothenberg, D.: A Model for Pattern Perception with Musical Applications. Part I: Pitch Structures as Order-Preserving Maps. Mathematical Systems Theory 11, 199-234 (1978a)

11. Rothenberg, D.: A Model for Pattern Perception with Musical Applications. Part II: The Information Content of Pitch Structures. Mathematical Systems Theory 11, 353-372 (1978b)

12. Balzano, G.J.: The Group-Theoretic Description of 12-Fold and Microtonal Pitch Systems. Computer Music Journal 4(4), 66-84 (1980)

13. Carey, N.: On Coherence and Sameness, and the Evaluation of Scale Candidacy Claims. Journal of Music Theory 46(1/2), 1-56 (2002)

14. Block, S., Douthett, J.: Vector Products and Intervallic Weighting. Journal of Music Theory 38(1), 21-41 (1994) 